\title{
Confrontation Naming Errors of Alzheimer's Disease Patients
}

\author{
Stephen C Enwefa* and Regina Enwefa \\ Department of Speech Language Pathology, Southern University and A \& M College, USA
}

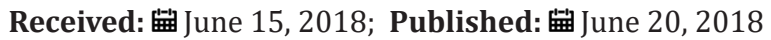

*Corresponding author: Stephen C Enwefa, Department of Speech Language Pathology, College of Nursing and Allied Health, Southern University and A \& M College, Baton Rouge, LA, USA

\begin{abstract}
This study investigated confrontation naming errors of Alzheimer's disease patients. Clinicians lack a validated test battery for differentiating the communication disorders of patients with AD from either normal elderly or patients with aphasia [1,3]. The communication of $\mathrm{AD}$ patients is often assessed with one of the standardized test batteries for aphasia. This was done because of the marked discrepancy between language and other cognitive functions. A linguistic measure involving errors in confrontation naming was used to establish the extent of linguistic impairment of AD patients. A total of ten photographs were shown to twenty $\mathrm{AD}$ patients, (ten mild and ten moderate) and ten normal elderly. The results showed that naming errors increased as the disease progressed. The study concluded that the number of naming errors of AD patients increased as the severity of the disease progressed.
\end{abstract}

\section{Introduction}

Alzheimer's disease (AD) is one of the most common and dreaded diseases afflicting the elderly community of all ethnic races. It does not discriminate, nor is it only found in certain age groups. $\mathrm{AD}$ affects all social, ethnic, racial, economic groups around the world. AD is no longer an old age problem. AD was first described by Alois Alzheimer in 1906 as a presenile dementia. AD is characteristics by a wide range of clinical disorders. Approximately 5.4 million Americans now have AD with a projected increase to 16 million by 2050 [2]. About one in every three seniors dies of AD or another dementia. AD kills more than breast and prostate cancer combined [2].

$\mathrm{AD}$ is a neurodegenerative disease. It affects persons of sexes, races, and ethnic backgrounds. AD affects more women than men. The major risk factors for $\mathrm{AD}$ are age and heredity. Persons with a high incidence of $\mathrm{AD}$ in their family history are most vulnerable. However, it is a severely debilitating form of mental dementia that affects the individual, families, homes, communities, etc. Although there are some other forms of dementia, there is currently no cure for Alzheimer Disease. AD is the most common cause of dementia among people age 65 and older. By 2050, 13.8 million older people are expected to have AD if the current numbers hold and no preventive treatments become available [2]. The US Census Bureau suggests that the number may be a high as 16 million by the middle of the century. For every 5-year age group beyond 65 , the percentage of people with AD doubles. Five percent of people aged
65 to 74 and roughly 35 to 50 percent of people aged over 85 have the disorder, according to the Alzheimer's disease Association [2].

Today there are approximately 5.7 million people living with Alzheimer's disease [2]. 5.5 million are age 65 and older and 200,000 are under age 65 and have younger-onset Alzheimer's disease. Every 65 seconds someone develops Alzheimer's disease and by 2050 someone will develop the disease every 33 seconds. Research has shown that AD increases with age. It is projected that individuals 65 years and older will grow from 53 million in 2018 to 88 million by 2050 [3]. In 2011, baby boomers began turning 65 so one can see we are living longer. For future years to come, ten million baby boomers will develop Alzheimer's disease in the United States (1 out of 9)[2].

A general overview of Alzheimer's disease including the clinical description, diagnosis, and progression of symptoms, benefits one to further understand the treatment and care of patients, the scope of the problem, scope of practice, and the current research which is forever changing. Dementia and Alzheimer's disease may be equivalent in some ways. It is important to relate dementia and Alzheimer's disease to grasp the differences between the two conditions. Alzheimer's is the most common form of dementia marked by memory loss in older individuals. Dementia is the gradual loss of intellectual function. Nonetheless, Alzheimer's disease is not a normal part of aging and is not something that unsurprisingly happens in later life. Alzheimer's disease can be 
defined as a form of dementia characterized by a gradual loss of several important mental functions that interrupts the normal flow of life. It is perhaps, the most common cause of dementia in older Americans, and goes beyond just normal forgetfulness, such as losing your car keys or forgetting where you parked. Signs of Alzheimer's disease include language deficits, memory loss that is much more severe and more serious, such as forgetting the names of your children or perhaps where you've lived for the last decade or two, and remembering when you had your last meal.

Numerous studies have investigated naming errors in AD by classifying errors as visual, semantic or lexical in nature [4-6].

A common finding is that $\mathrm{AD}$ patients produce many semantic and/or thematic naming errors (i.e. shark for dolphin). The criteria by which errors are divided can potentially overlook interactions among perceptual and lexical-semantic processes.AD can be considered a multifocal disorder; one must consider the possibility that visual perception and naming are two unrelated areas of concomitant decline. However, there is evidence of a possible link between the two processes in visual perception tasks that require discrimination of real objects according to Tippett and Blackwood [7]. Studies have found that when healthy individuals underwent fMRI while performing a visual discrimination task between line drawings, they recruited relatively more anterior regions of the fusiform gyrus when the two drawings had high structural similarity and relatively more posterior regions of the fusiform gyrus and inferior occipital cortex when the drawings were lower in structural similarity. Together these findings pose that fMRI signal in mid-anterior areas are related to processing of detailed object structure because these regions are sensitive to pictures with high structural similarity and pictures that require high specificity of structural processing, but not to other types of visual similarity according to Bussey and Saksida [8]. Damage to this common neural substrate devoted to Hajilou and Done [9].

Visual discrimination tasks that fluctuate in the degree to which they likely access structural and semantic knowledge could possibly provide awareness into visual perceptual or object recognition deficits in $\mathrm{AD}$ that may impact picture naming errors [10-12]. Visual discrimination tasks that require matching of complex shapes should not depend on access to a structural description system. Visual discrimination tasks should help guide the process of discrimination. Visual discrimination tasks that require an individual to determine if line drawings of real objects are of the same or different object in different views require accurate low level visual perceptual processing and reference to a stored structural representation to help guide the decision.

There are a number of potential ways that visual perceptual impairment may impact lexical-semantic processing in AD. Visual deficits may impact confrontation naming because of weakened visual input $[13,14]$.

Picture naming errors was used to study language deficits in Alzheimer's disease (AD) patients. Confrontation naming difficulties confounds performance on a memory test $[4,10]$. Picture naming tests have been extensively used to study cognitive and language deficits in $\mathrm{AD}$. $\mathrm{AD}$ patients picture naming errors increases as a result of the disease severity $[5,14]$.

Also, the number of pictures $\mathrm{AD}$ patients can name and the type of naming errors changed as the disease progresses and determined that confrontational naming errors of AD patients increased based on the stage of the disease. Assessment of a patient's cognition is a crucial part of many medical consultations. Cognitive tests aid the diagnosis of dementia and are important in the medical and social management of patients and in the assessment of capacity. Several studies have investigated naming errors in AD by classifying errors as visual, semantic or lexical in nature. A common finding is that $\mathrm{AD}$ patients produce many semantic and/or thematic naming errors (i.e. zebra for horse).

Evidence for a deterioration of semantic memory in AD comes from several studies that probed for knowledge of particular concepts across different modes of access and output (e.g., fluency, confrontation naming, sorting, word-to-picture matching, and definition generation). In assessing $\mathrm{AD}$ patients, there is a lack of specificity to test and identify AD. Before diagnosis of the disease is made, other conditions must be excluded, such as depression, adverse drug reactions, metabolic changes, nutritional deficiencies, chemotherapy, head injuries, and stroke.

The scientific community is applying the newest knowledge and research techniques in molecular genetics, pathology, virology, immunology, toxicology, neurology, psychiatry, pharmacology, biochemistry, and epidemiology to determine cause, diagnosis, treatment, and cure for AD.

The current practice is to use the DSM-V; and there has been considerable debate as to the diagnostic criteria. AD is now classified as Major and Minor Neurocognitive Disorders. The term Dementia has been removed. The diagnosis of dementia corresponds to a variety of etiological domains, from cerebrovascular disease to neuro degenerative disease. However, Alzheimer's Disease (AD), which is a neurodegenerative disease of uncertain origin and pathogenesis,is the most shared form of dementia in the elderly. While the National Institute of Neurological and Communicative Disorders and Stroke and the Alzheimer's Disease and Related Disorders Association(NINCDS-ADRDA)NINCDS-ADRDA guidelines have been used for a long time and have been shown to have reasonable validity and reliability, they are lacking in specificity, and need to be updated to incorporate the latest advances indiagnostic technology. The progression of AD clinically is often measured by mental status scales such as the Mini-Mental State Examination (MMSE) and the Clinical Dementia Rating Scale. However, these scales have their own limitations in terms of corresponding with the rate of clinical decline.

\section{Method}

Clinicians lack a validated test battery for differentiating the communication disorders of patients with AD from either normal elderly or patients with aphasia $[15,16]$. The communication 
of $\mathrm{AD}$ patients is often assessed with one of the standardized test batteries for aphasia. This was done because of the marked discrepancy between language and other cognitive functions. This study determined whether the number of cognitive errors of $\mathrm{AD}$ patients on visual recognition tasks increases across cognitive tasks as the disease progresses. Secondly, to determine whether Visual confrontation naming errors increase based on stage of the disease.AD participants were all medically diagnosed with probable AD according to NINCDS and ADRDA. Participants with any form of vascular dementia, head trauma, alcoholism, depression, chemotherapy, or psychiatric illness were excluded from the study. A linguistic measure involving errors in confrontation naming was used to establish the extent of linguistic impairment of AD patients. A total of ten photographs were shown to twenty AD patients, (ten mild and ten moderate) and ten normal elderly. Age range was from 72-93 years of age. AD patients were given:10 real objects, 10 colored photographs of the real objects, and 30 colored photographs of any household object such as: spoon, cup, toothbrush, comb, etc. Visual Recognition Tasks (VRTs) was scored as follows: 1 correct response, 0 incorrect responses, NR no response. The naming phase was measured as follows: correct name of object, incorrect name of object, and no response.

\section{Results}

Descriptive Statistics were used to identify group means and standard deviations for all groups. Statistical comparisons of cognitive abilities between the overall performances of the groups were analyzed using ANOVA. Statistical differences were found between the group means of confrontation naming $(F(2,27)=$ 34.406, $\mathrm{p}<0.0001$ ).

\section{Conclusion}

The results showed that naming errors increased as the disease progressed. The study concluded that the number of naming errors of AD patients increased as the severity of the disease progressed. Since the visual discrimination task used real, nameable objects, it may have been a strong predictor of naming and semantic association abilities by tapping into a visual process that occurs at a stage prior to naming, such as comparing visual percepts to stored structural representations.

More data needed with respect to the ways in which cognitive deterioration in $\mathrm{AD}$ patients may be assessed during the staging of the disease. Future research should include other population samples of AD. This would aid in knowledge as to the variable features of AD. Longitudinal studies to determine the distinctive elements of $\mathrm{AD}$, rates of decline, and reliable staging techniques. More time is needed to assess the new DSM-V criteria for AD patients. Our findings suggest that there is a common deficit in discrimination of pictures using nameable objects, picture naming and semantic association of pictures in AD. Future studies may investigate whether a treatment approach targeting visual discrimination abilities improves lexical-semantic performance in this population.

\section{References}

1. Alzheimer's Association (2018) Alzheimer's Disease Facts and Figures. Alzheimers Dementia 14(3): 367-429.

2. Alzheimer's Disease Association (2016) AD Facts and Figures: Alzheimer's Association Report, Chicago, IL, USA.

3. He W, Goodkind D, Kowal P (2016) An Aging World: 2015, International Population Reports. U.S. Government Publishing Office, Washington, USA 95: 16-1.

4. Enwefa R, Enwefa S (2006) Screening Alzheimer's Disease Patients for Subtle Deficits. National Association of African American Studies Health Monograph Series. University of New England, Biddeford, ME, England.

5. Enwefa R, Enwefa S (2006) Projections of Alzheimer's Disease (AD) in United States Latinos/Hispanics Populations. National Association of African American Studies Health Monograph Series. ME: University of New England, Biddeford, England.

6. Enwefa S, Enwefa R (2005) Alzheimer's disease in African Americans. Proceedings of the Annual Conference, Mississippi Speech-LanguageHearing Association, Jackson, MS, USA.

7. Tippett L, Meier S, Blackwood K, Diaz Asper C (2007) Category Specific Deficits in Alzheimer's Disease: Fact or Artefact? Cortex 43(7): 907-920.

8. Bussey TJ, Saksida LM, Murray EA (2002) Perirhinal cortex resolves feature ambiguity in complex visual discriminations. European Journal of Neuroscience 15(2): 365-374.

9. Hajilou BB, Done DJ (2007) Evidence for a dissociation of structural and semantic knowledge in dementia of the Alzheimer type (DAT). Neuropsychologia 45(4): 810-816.

10. Enwefa S (2002) The effect of Alzheimer's Disease on Language. Journal of the National Society of Allied Health 1(2): 50-54.

11. Enwefa S, Enwefa R (2004) Alzheimer's disease in African Americans. The ASHA Leader 9(8): 8.

12. Enwefa S, Enwefa R (2004) Alzheimer's disease in African Americans: A Preliminary Report of a Quiet Epidemic. Journal of the National Society of Allied Health 2(3): 25-31.

13. Done DJ, Hajilou BB (2005) Loss of high-level perceptual knowledge of object structure in DAT. Neuropsychologia 43(1): 60-68.

14. Enwefa SC, Enwefa RL (2001) A Study of Language and Cognitive Functions in Alzheimer's disease Patients. Journal of Public Affairs.

15. Enwefa S, Enwefa R (2005) Subtle Language Deficits as a Marker of Alzheimer's disease (AD). International Week Symposium Monograph Series. Jackson, MS: Jackson State University, USA.

16. Bayles K, Boone D, Tomoeda C, Slauson T (1989) Differentiating Alzheimer's from the normal elderly and stroke patients with aphasia. Journal of Speech and Hearing Disorders 54(1): 74-87. 


\section{(C) Commons Attribution 4.0 License}

To Submit Your Article Click Here:

Submit Article

DOI: $10.32474 / 0 J N B D .2018 .01 .000117$

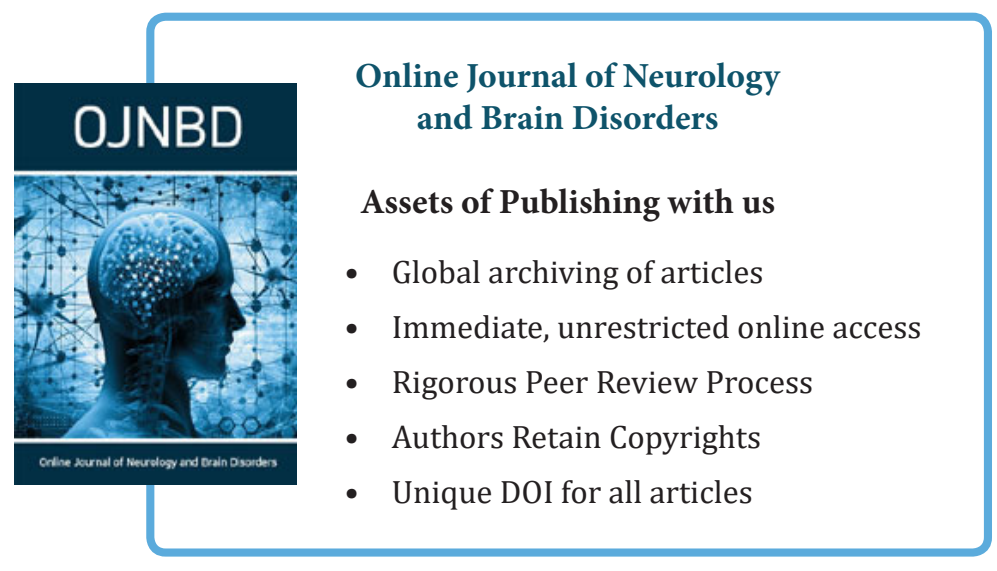

